\title{
DEVELOPMENT OF NEW SYNTHESIS ROUTES FOR HYDROXYAPATITE NANOPOWDERS PRODUCTION FROM CHICKEN EGGSHELLS
}

\author{
Ingrid Russoni de Lima ${ }^{\circledR a}$, , Bonifácio Oliveira Fialho ${ }^{a}$, Renata Antoun Simão ${ }^{\text {}}$, Daniele Aparecida Nogueira ${ }^{a}$, Fernanda \\ Aparecida Sampaio ${ }^{a}$, Yutao Xing ${ }^{c}$, Jose Adilson de Castro ${ }^{a}$, Gláucio Soares da Fonseca and Marcos Flávio de Campos ${ }^{a}$ \\ aDepartamento de Engenharia Metalúrgica e de Materiais, Universidade Federal Fluminense, 27255-125 Volta Redonda - RJ, Brasil

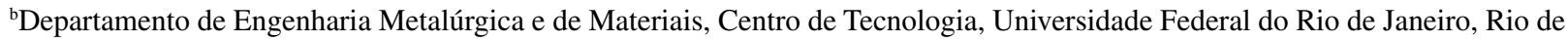 \\ Janeiro - RJ, Brasil \\ 'Departamento de Física, Instituto de Física, Universidade Federal, Fluminense, 24210-346 Niterói - RJ, Brasil
}

Recebido em 12/01/2021; aceito em 11/05/2021; publicado na web em 04/06/2021

\begin{abstract}
Hydroxyapatite (HA) nanopowders are attracting interest for surface application to ceramic biomaterials. In this study, we developed innovative and new viable synthesis routes of calcium phosphate nanopowders from the natural calcium carbonate of chicken eggshells. Following the four routes, alternative co-precipitation, vacuum process, brushite, and calcination, we obtained the HA samples, which were then characterized by different methods. The X-ray diffraction /Rietveld method showed the HA phases, monetite and brushite crystallinity with greater symmetry, and nanoscale. Transmission electron microscopy showed the morphology of nanoparticles functionalized nanoparticles smaller than $100 \mathrm{~nm}$ in routes A, B and D, and Fourier-transform infrared spectrophotometry and Raman spectroscopy identified the classic chemical groups of calcium phosphates by four routes mainly groups $\left(\mathrm{PO}_{4}\right)^{3-}$ and $\left(\mathrm{CO}_{3}^{-}\right)$. Scanning electron microscopy analysis revealed a good surface topography to future cell biointeraction in A, B and D routes, and thermogravimetric analysis showed the mass loss profile inherent to each synthesis route with slot at $200{ }^{\circ} \mathrm{C}$. Hence, the eggshell HA and brushite and the specific monetite nanoparticles with different morphology can be prepared from the four new possible and viable routes using different methods.
\end{abstract}

Keywords: new synthesis routes; eggshell hydroxyapatite; brushite; physical-chemical characterization.

\section{INTRODUCTION}

Hydroxyapatite (HA), $\mathrm{Ca}_{10}\left(\mathrm{PO}_{4}\right)_{6} \mathrm{OH}$, belongs to the class of biomaterials based on calcium phosphate and presents physicalchemical properties suitable to bone loss replacement due to accidents or pathologies and in critical defects of human bone. HA's biocompatibility, moldability, and stability are likely to be similar to human bones and dental tissues. ${ }^{1}$ Calcium phosphate is a family of materials and minerals containing calcium ions $\left(\mathrm{Ca}^{2+}\right)$ together with orthophosphates $\left(\mathrm{PO}_{4}{ }^{3-}\right)$, metaphosphates, or pyrophosphates $\left(\mathrm{P}_{2} \mathrm{O}_{7}{ }^{4-}\right)$, and sometimes hydrogen or hydroxide ions. ${ }^{2} \mathrm{HA}$ is the major inorganic component of natural bone, which can be obtained synthetically at the laboratory level. HA with calcium phosphate also participates in the therapy of several pathologies, such as treatments of osteonecrosis, lesions, and actinic purpura, to fill and promote the repair process of tissue defects. ${ }^{3}$

There are several methods in obtaining advanced ceramic (HA) from synthetic reagents, such as combustion, hydrothermal, and sol-gel methods. The most traditional and mostly used HA synthesis is the co-precipitation method because of its viability, control of stages, and simplicity, as compared with other methods. ${ }^{4}$ Another advantage of this method is that it uses natural precursor reagents instead of synthetic reagents. Besides, it is the current alternative method when considering sustainability. ${ }^{5}$ Synthesis by wet precipitation or co-precipitation is a viable and low-cost methodology in obtaining ultra-fine powders and even nanometric powders in traditional synthesis. ${ }^{6}$ Although other routes have been developed and elaborated, such as synthesis by hydrothermal process, sol-gel methods in aqueous or alcoholic medium, crystallization, the wet precipitation method is still the most widely

*e-mail: ingridrussoni@gmail.com used because of its feasibility and simplicity in obtaining products with profiles similar to human bones and dental tissues. Thus, HA with calcium phosphate and advanced ceramic, HA from synthetic reagents with a great value of the final product, we developed a new stoichiometric synthesis and applied the four syntheses from a natural reagent. Based on the classic route for obtaining hydroxyapatite by co-precipitation and chemical reaction, this route obtains reagent naturally, which is different from traditional routes and routes still in development. HA can be synthesized naturally from different calcium phosphate sources, such as the inorganic part of human bones and living tissues, and other sources. ${ }^{7}$ Natural carbonates are materials found in bovine bones, shells, and crustaceans. ${ }^{8,9}$ Obtaining hydroxyapatite from natural reagents is still very challenging, and using an efficient biomaterial in practical use is still far from being applied in humans because of many patent secrets inherited in the biomaterials applied in clinical practice.

Because of their mineralogical characteristics, these natural origin carbonates have been presenting themselves as favorable raw materials in elaborating biomaterials, mainly in the synthesis of HA and calcium phosphates. ${ }^{10}$ Chicken eggshells are a rich source of calcium oxide that can function as precursors in the synthesis of calcium phosphates, especially HA. ${ }^{11}$ However, obtaining nanopowders from established synthesis is an enormous challenge. The eggshells from chickens and quails become a very attractive source material because of calcium carbonate calcium oxide, which can be used as a precursor to HA production. ${ }^{12,13}$ Calcium oxide can be obtained by chemical or calcined route, thus providing a natural low-cost, sustainable, and viable precursor reagent and saving some steps on average in synthesis processes, reducing the synthesis process. Thus, obtaining hydroxyapatite nanopowders (advanced ceramic) synthesis routes from natural reagents with significant characterization and functionality perspective results 
is still a challenge in the scientific community. Besides, there is a challenge in synthesizing, obtaining, and characterizing using different methods and then proving the comparison results obtained in high symmetry materials to obtain the nanopowdersIn this study, we developed and established new innovative routes for obtaining HA and brushite nanopowders from eggshells using many innovation processes (including innovation in different process steps) to each route to demonstrate its technical feasibility. The new three routes for HA fabrication used different precursors and calcium oxide from eggshells as the source material. The results were compared with the literature's traditional methods; however, we modified the literature's adapted protocol base. The advantage of developing this methodology is the re-use of waste that would naturally be disposed of in landfills producing valuable products that will be properly processed in line with the development of biomaterials. ${ }^{14}$ The nanopowders are attracting interest for surface application to biomaterials because they are more viable, low costs, and better biocompatibility in interacting with biological fluids, ensuring better functionality with the prospect of patents or contributing better surfaces of future biomaterials to be disposed of in the national health system. Hydroxyapatite is the most biocompatible ceramic biomaterial. Brushite has several applications and it is already used as biomaterial; moreover, it is a more soluble calcium phosphate, which is interesting for applications, especially in biomaterials' surface coating. This paper presents many scientific contributions in the development of new routes of nanopowders obtained from stoichiometric specifics.

The objectives of this study were as follows: (i) to determine new routes with specific and controlled steps in obtaining hydroxyapatite nanopowders from chicken eggshells with specific stoichiometry; (ii) to demonstrate that the calcium oxide reagent can be obtained chemically and then compare with the modified calcined route (route D) balanced equation; (iii) to obtain nanopowders of different proportions of phases and specific steps in the protocol and then optimize them after performing several experiments, including the development and application of own drippers developed during the syntheses, the consolidation by different techniques of specific characteristics that include the sum of acquired data, and the comparison of the routes from the same analysis protocols and conditions, and (iv) to find the HA, monetite, and brushite nanopowders with higher symmetry structure than that usually reported in the literature.

\section{MATERIAL AND METHODS}

\section{Materials}

In this study, we designed and developed new nanopowders synthesis and then characterized the obtained hydroxyapatite with calcium phosphate, calcium phosphate, brushite and monetite using the development of four routes. ${ }^{15}$ We used non-free-range chicken eggshells from a standard farm (Granja Rica, Pinheiral City). The eggshells rich in calcium carbonate were washed with the following: detergent and sponge, current water, running distilled water for more than 20 times, and subsequent bath of distilled water four times. The clean eggshells were dried in an oven at $50^{\circ} \mathrm{C}$ for $12 \mathrm{~h}$ and then crushed in agate grits in intermittent movements to directly obtain the precursor powder from the eggshell. The objective that no calcined the three first routes was to use the lowest energy needed. Calcined step demands calcination would lead to an unnecessary amount of energy. So, only the fourth route was calcinated and we managed to compare the non-calcined routes with the last calcined route. Other chemical reagents used in different routes were obtained commercially.

\section{Processing}

To obtain the calcium phosphate, we developed four routes: routes $\mathrm{A}-\mathrm{C}$ were newly developed, and route $\mathrm{D}$ was using the same protocol from the literature. Details of the four different routes are discussed in the following.

\section{Synthesis routes according to the type of hydroxyapatite selected}

Route A. The initial objective of this route was to produce calcium phosphate by "alternative co-precipitation." In this new route elaborated in our laboratory, the $\sim 10,0 \mathrm{~g}$ eggshell powder was processed and then added into a $400-\mathrm{mL}$ beaker together with $\sim 10$-mL glacial acetic acid (P.A. 1 L, CAS, 64-19-7). The beaker was filled with $400 \mathrm{~mL}$ distilled water, and the mixture was magnetically stirred for $4 \mathrm{~h}$. The eggshells were dissolved after $12 \mathrm{~h}$, and the solution was filtered to remove the solid residues. Approximately $50 \mathrm{~mL}$ of $2 \mathrm{~mol} \mathrm{~L}^{-1}$ phosphoric acid was added to the filtrate, and the solution was stirred and heated at $50{ }^{\circ} \mathbf{C}$ for $2 \mathrm{~h}$. After decanting the supernatant, the precipitated solids were washed with distilled water three times and always put in agitation at $50{ }^{\circ} \mathrm{C}$. After washing, the obtained precipitate was placed in an oven at $40{ }^{\circ} \mathrm{C}$ for drying.

Route B. This route, the "vacuum process," aimed to obtain calcium phosphate hydroxyapatite from the eggshells.

Among the possible acids that could be used for eggshell dissolution, we chose the acetic acid (P.A. 1 L, CAS, 64-19-7). Other acids, such as hydrochloric acid, could hinder the phosphate's subsequent precipitation because of the high solubility of the calcium chloride formed. However, sulfuric acid could result in impurities from the reaction with organic compounds.

After it has stayed overnight, the eggshells had not been dissolved completely; considering the Chatelier Law, the reaction speed depends on the concentration of the starting reagents, in this case, as carbon dioxide is a by-product of the reaction when the solution is saturated, the solution is fixed in $\mathrm{pH}$ from this point on, so, the solution was still acidic, with a $\mathrm{pH}$ of $\sim 4.5$. As the reaction releases carbon dioxide $\left(\mathrm{CO}_{2}\right)$, it was assumed that the resulting solution was saturated with that gas, thus interfering with the reaction balance. Hence, approximately half of the supernatant was transferred to another beaker, which was identified as experiment $\mathrm{A}$, and the rest was identified as experiment B.

Experiment A was vacuum filtered on a quantitative filter paper $\left(85 \mathrm{~g} / \mathrm{m}^{2}\right)$ using the filtration vacuum to remove the dissolved carbon dioxide. After filtration, $100 \mathrm{~mL}$ of $2-\mathrm{mol} \mathrm{L}^{-1}$ phosphoric acid was added, and the solution was heated at $60{ }^{\circ} \mathrm{C}$ with stirring for $4 \mathrm{~h}$. After the precipitate was formed, the stirring was stopped, and the precipitate was expected to decant. After settling the precipitate, ammonium hydroxide was added until the supernatant reached close to $\mathrm{pH} 10$. The system was stirred again for $\sim 0$ min while keeping the temperature at $\sim 60{ }^{\circ} \mathrm{C}$. The supernatant was discarded after the precipitate was decanted. The obtained precipitate was washed in distilled water three times while keeping the temperature at $\sim 60{ }^{\circ} \mathrm{C}$ and then stirred for a few minutes. After washing, the obtained precipitate was placed in an oven at $40{ }^{\circ} \mathrm{C}$ for drying during the night.

In parallel, we completed the beaker's volume in experiment B to be $600 \mathrm{~mL}$ again to dilute the system. The obtained suspension (still containing fragments of eggshells) was vacuumed to remove the dissolved carbon dioxide, displace the reaction balance, and complete the dissolution of the remaining shell fragments. The system was also placed under agitation to accelerate the dissolution process. After the eggshells were dissolved completely, the solution 
went through the same procedure described for experiment $\mathrm{A}$. The difference between the experiments was essential in the initial concentration of the solution, which resulted from the dissolution of the eggshells: experiment A started with a more concentrated solution of the calcium acetate.

Route C. In this route, "brushite," we used the same reagents described above and added a new step preceded by $\mathrm{NaOH}$. We packed $20 \mathrm{~g}$ of eggshell powder into the beaker and then added $30 \mathrm{~g}$ of glacial acetic acid. The beaker was filled with $600 \mathrm{~mL}$ distilled water, stirred to mix the components, and left to stand for $\sim 17 \mathrm{~h}$ to dissolve the eggshells. Similar to the results in route B, the dissolution of the shells was not completed; hence, vacuum treatment is needed to remove excess $\mathrm{CO}_{2}$ in the solution.

After the shells' dissolution was completed, we filtered the solution through a quantitative filter and added, by stirring, small quantities of sodium hydroxide until it reached close to $\mathrm{pH} 10$.

Thus, we obtained a gelatinous precipitate in the suspension, and while stirring, we added $100 \mathrm{~mL}$ of $2-\mathrm{mol} \mathrm{L}^{-1}$ phosphoric acid. After a few minutes of stirring, the precipitate's appearance became pulverized, and after stirring, it became decanted. Similar occurrences of the precipitate are obtained in route B. After decanting, the supernatant was discarded, and the precipitate was washed three times (the same in previous procedures), except that the whole process was performed at room temperature.

Route D. Natural reagents were used together with the traditional method with calcination. In route $\mathrm{D}$, it started based on the work by Farias ${ }_{15}^{15}$ adapting the original protocol and modifying the parameters. The specific protocol observed is that the $\mathrm{CaO}$ is obtained by calcination ramp. Here, the eggshells were crushed and took the ramp at $800{ }^{\circ} \mathrm{C}$ for $3 \mathrm{~h}$, thus obtaining the $\mathrm{CaO}$. We obtained the dripping of distilled water with a specific mixture, which is the $\mathrm{Ca}(\mathrm{OH})_{2}$. (The dripping is planned according to the stoichiometry of phosphoric acid for $24 \mathrm{~h}$.) It was then oven-dried at $50{ }^{\circ} \mathrm{C}$ overnight and then calcined for $1100^{\circ} \mathrm{C}$ using the specified ramp [not being an exact reproduction of Farias' work ( $900{ }^{\circ} \mathrm{C}$ for $2 \mathrm{~h}$ ) because of minor modifications to the initial protocol].

\section{Physico-chemical characterization of hydroxyapatites based on calcium phosphate}

X-ray diffraction (XRD). We used XRD to characterize the phases and the crystallinity of carbonate, phosphate, and hydroxyapatite powders synthesized using the four routes. The analysis was performed on a Bruker D8 Advance diffractometer (Bruker Co., Billerica, MA, USA) with $\mathrm{Co}-\mathrm{K} \alpha$ radiation source, capillary measurement accessories, spinner, and a LynXeye rapid detector in the XRD laboratory at the Institute of Physics of Niterói UFF. The analyses were also performed in a scan range from $10^{\circ}$ to $80^{\circ}$ with a step size of $0.02^{\circ}, 0.5 \mathrm{~s}$ for each step, and $15 \mathrm{rpm}$ for the sample holder rotation. The final interpretation was made by Rietveld XRD analysis.

\section{Rietveld analysis (specific XRD analysis)}

We used the TOPAS-Academic software (Coelho Software, Brisbane, Australia). ${ }^{16}$ The main constituents, of the samples are hydroxyapatite, calcium phosphate, monetite and brushite (the space group number SG of the phases is given along this study). The curved shape of the diffractogram baselines is due to the amorphous filter paper. As amorphous phases generate no diffraction peaks, the Rietveld method treats the sample as if it only contains crystalline phases. In other words, the background of the x-Ray diffraction spectra can be described by a function in the Rietveld software (as for example a polynomial). It was found that it is possible to describe the brushite with a structure of greater symmetry that that commonly reported on literature.

Transmission electron microscopy (TEM). We used TEM to investigate the detailed microstructure, morphologies, and particle size of the powders obtained using the four routes at the LaMAR/ CAIPE-UFF. The high-resolution (HR) TEM (JEOL JEM-2100F) operates at $200 \mathrm{kV}$ with a field emission gun. The samples for TEM analysis were prepared by mixing the powders with ethanol and then dropping a small amount of the clear upper-part liquid to the $\mathrm{Cu}$ grids with a thin layer of $\mathrm{C}$ support (3-5 nm). After drying completely, we ready the $\mathrm{Cu}$ grids for TEM analysis.

Scanning electron microscopy (SEM). We used SEM to analyze the surface structure because there was a need to simulate a surface of possible interaction with the cells. Thus, the powders of hydroxyapatite tablets' pastilles were synthesized and then were subjected to uniaxial pressing at $500 \mathrm{kgf}$ and axially incident (uniaxial pressing of the powders); meanwhile, 12-mm-diameter steel matrix was used for measuring the tablets in $12-\mathrm{mm}$ diameter. In every pressing, there was lubrication with oleic acid after the cleaning. Each tablet's uniaxial pressing time was fixed at $1 \mathrm{~min}$, thus obtaining the hydroxyapatite pastilles standard.

The samples were metalized in stubs using Emitech metallizer (K550X; Emitech, Dubai, UAE), under 50-mA current for $180 \mathrm{~s}$. Microstructural analyses were performed in SEM using the SEM IVO MA 10 Zeiss electron microscope (Zeiss, San Diego, CA, USA). The operating parameters used were 14- to 15-mm working distance. The analyses were performed in the SEM laboratory (SEM-UFF Volta Redonda, RJ, Brazil) for the topographic characterization and particle morphology by SEM.

Fourier-transform infrared spectroscopy (FTIR). We used FTIR to characterize the vibrational bands of the $\mathrm{CO}_{3}{ }^{2-}, \mathrm{PO}_{4}{ }^{3-}$, and $\mathrm{OH}^{-}$groups that normally occur in the hydroxyapatite standard (as a kind of fingerprint of the chemical compound). This analysis used the Perkin-Elmer Spectrum (PerkinElmer, Waltham, MA, USA) with attenuated reflectance, using the range from 4000 to $400 \mathrm{~cm}^{-1}$ with an accuracy of $4.00 \mathrm{~cm}^{-1} .{ }^{17}$ This technique has been widely used to identify the chemical groupings of chemical elements by studying the vibration bands of present chemical bonds in the molecules, thus building bases for identifying chemical molecules by identifying key elements present and allowing the identification inherent to each specific molecule that translates the existence of the chemical compound. $^{18}$

Raman spectroscopy (RS). Raman spectroscopy is a highresolution photonic technique that can provide chemical and structural. The technique is based on laser light, monochromatic, collimated, coherent and of a certain frequency, spread when focusing on the material to be studied, whose majority of the scattered light also has the same frequency as that incident. Only a small portion of the light is scattered inelastically in the face of rapid changes in frequency, due to the interaction of light with matter, and is an intrinsic characteristic of the material analyzed and does not depend on the frequency of the incident light. The most important is the inelastic effect also known as the Raman effect to obtain many important information about the chemical composition that generates a signature of the chemical composition (fingerprint). When the sample analyzed generates fluorescence, there may be a fluorescence noise effect (photon noise or shot noise), which can cause data loss or even make it impossible to identify essential groups using the Raman technique. We performed Raman spectroscopy (RS) analysis using RamanWitec focus innovations (alpha 300; Coppe/UFRJ, Rio de Janeiro, Brazil) operating at a 785-nm wavelength and maximum output laser intensity. During operation, the pinhole diameter was $100 \mu \mathrm{m}$ with an 
integration time of $50 \mathrm{~ms}$, and a grating of $600 \mathrm{~g} / \mathrm{mm}$ in a complete protocol established by Raman's laboratory. ${ }^{19}$ The routes' powders were analyzed using Raman-Witec focus innovations (Coppe/UFRJ).

Thermogravimetric analysis (TGA). We performed TGA analysis on the different synthesized powders obtained by synthesizing green co-precipitation using SDT-600 (Steel Dragon, Mayangone, Myanmar). It was analyzed in a range from $25^{\circ} \mathrm{C}$ to $1000{ }^{\circ} \mathrm{C}$ in air and a heating rate of $15^{\circ} \mathrm{C} .{ }^{20}$ The isotherms were drawn to determine the loss of mass of each synthesis and condition, thus comparing the processes among themselves. In TGA, the powders were analyzed at the Universidade Federal Fluminense (Volta Redonda, Brazil) for a postgraduate engineering course. Moreover, TGA is widely used to evaluate degradation, dehydration, swelling, and mass loss.

\section{RESULTS AND DISCUSSION}

The results showed that it was possible to obtain the four specific reaction describe bellow:

Route $A$ - It was possible to obtain calcium phosphate by "alternative co-precipitation."

$$
\mathrm{CaCO}_{3(\mathrm{~S})}+2 \mathrm{H}_{(\mathrm{Aq})}^{+} \rightarrow \mathrm{Ca}^{2+}{ }_{(\mathrm{Aq})}+\mathrm{H}_{2} \mathrm{O}_{(\mathrm{L})}+\mathrm{CO}_{2(\mathrm{G})}
$$

Route B - This route, the "vacuum process," aimed to obtain calcium phosphate hydroxyapatite from the eggshells, as described in the following.

$$
\mathrm{CaCO}_{3(\mathrm{~S})}+2 \mathrm{H}^{+}{ }_{(\mathrm{Aq})} \rightarrow \mathrm{Ca}^{2+}{ }_{(\mathrm{Aq})}+\mathrm{H}_{2} \mathrm{O}_{(\mathrm{L})}+\mathrm{CO}_{2(\mathrm{G})(\text { vacuum })}
$$

Among the possible acids that could be used for eggshell dissolution, we chose the acetic acid (P.A.1 L, CAS, 64-19-7) because it is a moderately strong acid, and the calcium acetate, which is the product of the reaction, is very soluble. Other acids, such as hydrochloric acid, could hinder the phosphate's subsequent precipitation because of the high solubility of the calcium chloride formed. However, sulfuric acid could result in impurities from the reaction with organic compounds

Route C. In this route, "brushite," we used the same reagents described above and added a new step preceded by $\mathrm{NaOH}$. The calcium hydroxide started to precipitate, according to the following reaction:

$$
\mathrm{Ca}(\mathrm{Ac})_{2}+2 \mathrm{NaOH} \rightarrow \mathrm{Ca}(\mathrm{OH})_{2}+2 \mathrm{NaAc}
$$

Route $D$. Natural reagents were used together with the traditional method with calcination. It was possible to obtain $\mathrm{CaO}$ using using specific calcinated ramp from Farias protocol.

\section{X-ray diffraction}

Figures 1a-1d shows the Rietveld analysis following the four routes, and Table 1 gives the phase quantification. The structural data of the phases is presented in Tables 2-4.

A given structure is described by three lattice parameters a,b,c and by three angles the angles $\alpha, \beta, \gamma$. Phases with high symmetry - as cubic - only need the lattice parameters for structure description. For example the angles $\alpha=\beta=\gamma$ f are identical for a cubic phase, and are $90 \mathrm{o}$ and also $\mathrm{a}=\mathrm{b}=\mathrm{c}$. Thus, the value of the a parameter is sufficient for describing a cubic phase with high symmetry (as for example fcc nickel). However, for phases with low symmetry as brushite and monetite the low symmetry implies that a,b,c may be different and also the angles $\alpha, \beta, \gamma$ may be different.
SG is abbreviation of Space Group. Each Space Group denotes a different structure, SG varies between 1 to 230. For example, the structure 229 is for high symmetry cubic bcc (as alpha-iron). The structure 225 is for fcc metals (as nickel, copper, aluminum).

The three phases identified in the samples are hydroxylapatite $(\mathrm{SG}=176)$, monetite $(\mathrm{SG}=2)$ and brushite $(\mathrm{SG}=15)$. Hydroxyapatite has high symmetry, $\mathrm{a}=\mathrm{b}$, and $\mathrm{c}$ is different from $\mathrm{a}$. Concerning the angles, for $\mathrm{SG}=176, \alpha=\beta=90^{\circ} \gamma=180^{\circ}$. Thus, angles are not displayed in Table 2.

Table 1. Phase quantification

\begin{tabular}{cc}
\hline Sample & Phase quantification \\
\hline A & $10 \%$ monetite $90 \%$ HA \\
B & $42 \%$ brushite $58 \%$ HA \\
C & $100 \%$ brushite \\
D & $46 \%$ monetite $54 \%$ HA \\
\hline
\end{tabular}

Table 2. Rietveld refinement results for lattice parameters for the HAP phase (hexagonal SG = 176)

\begin{tabular}{ccc}
\hline Route & a (Angstroms) & c (Angstroms) \\
\hline A & 9.447 & 6.873 \\
B & 9.471 & 6.888 \\
C & - & - \\
D & 9.428 & 6.897 \\
\hline
\end{tabular}

Table 3. Rietveld refinement results for lattice parameters and angles for the brushite phase $(\mathrm{SG}=15)$

\begin{tabular}{ccccccc}
\hline Route & $\mathrm{a}$ & $\mathrm{b}$ & $\mathrm{c}$ & alpha & beta & gamma \\
\hline A & & & & & & \\
B & 6.364 & 15.15 & 5.811 & 90 & 118.53 & 90 \\
C & 6.367 & 15.18 & 5.814 & 90 & 118.53 & 90 \\
D & & & & & & \\
\hline
\end{tabular}

Table 4. Rietveld refinement results for lattice parameters and angles for the monetite phase $(\mathrm{SG}=2)$

\begin{tabular}{ccccccc}
\hline Route & $\mathrm{a}$ & $\mathrm{b}$ & $\mathrm{c}$ & alpha & beta & gamma \\
\hline $\mathrm{A}$ & 6.897 & 6.636 & 6.991 & 96.35 & 103.90 & 88.73 \\
$\mathrm{~B}$ & & & & & & \\
$\mathrm{C}$ & & & & & & \\
$\mathrm{D}$ & 6.904 & 6.620 & 7.150 & 96.89 & 103.53 & 88.82 \\
\hline
\end{tabular}

As shown in Tables 2-4, the three phases observed in samples A, $\mathrm{B}, \mathrm{C}$, and D agree with the literature's experimental observations..$^{21-23}$ If the mean's $\mathrm{pH}$ is low, brushite and monetite can appear instead of hydroxyapatite HA. ${ }^{21}$ Among the possible acids that could be used for eggshell dissolution, we chose the acetic acid (P.A.1 L, CAS, 64-19-7) because it is a moderately strong acid, and the calcium acetate, which is the product of the reaction, is very soluble. Other acids, such as hydrochloric acid, could hinder the phosphate's subsequent precipitation because of the high solubility of the calcium chloride formed. However, sulfuric acid could result in impurities from the reaction with organic compounds.

The HA phase prototype is hexagonal and has a space group $(\mathrm{SG})=176 .{ }^{24}$ The prototype for the monetite is that given. Monetite DCPA CaHPO the low $\mathrm{SG}$ number $=2$, monetite is a phase with very low symmetry. 
The sample of route $\mathrm{C}$ is $100 \%$ brushite. Two different structures have been suggested for brushite: $\mathrm{SG}=9$ and $\mathrm{SG}=15$ (Ia or $I 2 / a$, respectively) ${ }^{26}$ If the structure was $S G=9$, then the reflection (110) should appear. As the reflection (110) is absent, the phase of sample C (brushite) belongs to $\mathrm{SG}=15$ and is in agreement with the structural data for brushite. ${ }^{27}$ Tables 3 and 5 give the structural data for sample C's brushite (100\% brushite).

Table 5. Structural data for brushite $I 2 / a(\mathrm{SG}=15)$

\begin{tabular}{cccc}
\hline \multirow{2}{*}{ Atom } & \multicolumn{3}{c}{ Experimental } \\
\cline { 2 - 4 } & $X$ & $Y$ & $Z$ \\
\hline $\mathrm{Ca}$ & 0.506 & 0.327 & 0.250 \\
$\mathrm{P}$ & 0.014 & 0.322 & 0.263 \\
$\mathrm{O}_{1}$ & 0.216 & 0.367 & 0.026 \\
$\mathrm{O}_{2}$ & 0.080 & 0.282 & 0.434 \\
$\mathrm{O}_{3}$ & 0.202 & 0.393 & 0.393 \\
$\mathrm{O}_{4}$ & 0.109 & 0.253 & 0.144 \\
$\mathrm{O}_{5}$ & 0.734 & 0.426 & 0.111 \\
$\mathrm{O}_{6}$ & 0.274 & 0.447 & 0.508 \\
\hline
\end{tabular}

There are some imperfections in the Rietveld adjusts. Rietveld analysis assumes only one crystallite size and only one lattice parameter. An additional difficulty for the Rietveld adjust is the off-stoichiometric behavior of the samples, especially for HA. The situation becomes even more complicated for phases with low crystalline symmetry, like the monoclinic. Other difficulties are that some samples are nanocrystalline, and peak overlap is significant.

Hydroxyapatite can be off-stoichiometric, resulting in calcium deficiency, with stoichiometry $\mathrm{Ca}_{10-x}\left(\mathrm{PO}_{4}\right)_{6-x}\left(\mathrm{HPO}_{4}\right)_{x}(\mathrm{OH})_{2-x}$. There are both anion $\left(\mathrm{OH}^{-}\right)$vacancies and cation vacancies $\left(\mathrm{Ca}^{2+}\right)$. Stoichiometric HA presents a $\mathrm{Ca} / \mathrm{P}$ ratio of $10: 6$ or 1.67 .

As shown in the TEM pictures (Figures 2a, 2b, 2d), HA's nanocrystalline grains are very anisotropic (two-dimensional needle shape). This is a problem for the Rietveld analysis because the grains rotate when the sample is pressed, generating a texture effect and affecting HA peaks, which is expressed as (hkl). Consequently, the (00l) peaks appear with higher intensity, whereas (hk0) peaks appear with reduced intensity. Because of this effect, the experimental intensity of the (2theta) peak near 300 [i.e., reflection (002)] for samples A and B is higher than the estimate obtained with Rietveld, as shown in Figure 1e.

Table 6 shows the crystallite size of HA estimated by Rietveld. The Rietveld estimates are in good agreement with the TEM observations (Figures $2 \mathrm{a}-2 \mathrm{~d}$ ). Thus, the objective of producing nanocrystalline HA was accomplished. The crystallite sizes of brushite and monetite are very high: above $150 \mathrm{~nm}$. The very thin peaks of brushite indicate crystallite size of the order of 1000 Angstroms or more.

Table 6. Crystallite size of hydroxyapatite (Rietveld estimated)

\begin{tabular}{cc}
\hline Route & crystallite size $(\mathrm{nm})$ \\
\hline A & 18 \\
B & 15 \\
D & 40 \\
\hline
\end{tabular}

The texture effect (as seen in Figure 1e) can be caused during the pressing of the milled samples for $\mathrm{x}$-Ray diffraction. This may happen when the crystals are strongly anisotropic, the case of HA, as shown in Figure 1e, which is based on Figure 1a. This effect reduces the intensity of (hk0) planes and increases the intensity of (001) planes, as shown in Figure 1e.

\section{Transmission electron microscopy}

In SEM analysis, it is difficult to see the individual particle size. Thus, we performed detailed studies of the HA samples obtained using the four routes with HR TEM, and the results are shown in Figure 2. In the Figure 2, the HA particles prepared by routes A and B present needle shapes with a length of higher than $100 \mathrm{~nm}$ and a width of $\sim 20 \mathrm{~nm}$. From the high-resolution image (Figures 2a1-2d1), we can observe that each needle is an HA single crystal. The larger particles with flat facets (Figure 2c) are not observed by TEM analysis because of sampling problems during the preparation of the TEM samples. [Only the upper (clear) part of the mixture (particles plus ethanol) were taken and dropped on the $\mathrm{Cu}$ grid.] The bigger particles stay in the lower (dark) part of the mixture and thus were not deposited on the $\mathrm{Cu}$ grid. We can conclude that particles with very different shapes and sizes were obtained by routes obtained. TEM pictures suggest a needle-like shape of HA particles. All HA particles' width and diameter are much lesser than $100 \mathrm{~nm}$, which is considered a key size for functionality.

Lattice parameters performed by Rietveld could identify the hydroxyapatite in the hexagonal structure with all the lattice parameters showed in Table 2 (X-ray diffraction Rietveld analysis). It was possible to analyze by Rietveld the monoclinic particles and estimate the nanocrystals' size in approximate line with the TEM data. Thus, Rietveld predicts crystallite sizes of $18 \mathrm{~nm}$ (synthesis A) with 20-nm width prediction by TEM, $15 \mathrm{~nm}$ (synthesis B) with 20-nm TEM width prediction, D synthesis with 40-nm range, and also for $\sim 20 \mathrm{~nm}$ by TEM.

\section{Scanning electron microscopy}

Considering that the proposed routes have the main objective of obtaining calcium phosphate powders in order to functionalize biomaterial surfaces, analyzing the surface topography by SEM is important in order to predict biocompatibility in relation to the most suitable topography. It was possible to analyze the surface of the four routes calcium phosphates. The interaction of the surface of the biomaterial is extremely important to modulate the interaction responses and modulation of the inflammatory and immune response of the human organism when interacting with the surface of the biomaterial, especially in the first 24-48 hours of biointeraction. Besides that, it was possible analysis for a suitable surface for cell interaction, being necessary biocompatibility analyses with cell culture proves in vivo this finding in vitro. ${ }^{28}$ On a smaller scale, the SEM images of green powders obtained after synthesis and drying in an oven at $50{ }^{\circ} \mathrm{C}$ overnight subjected to uniaxial pressing to form the tablets to focus on the surface. Hydroxyapatite was obtained in $a, b$, and $d$ conditions, and brushite plate was obtained in c condition. The SEM images in relationship to routes A-D are shown in Figures 3a-3d, respectively.

We can analyze SEM's morphological aspects similar tablets from the chicken eggs containing magnetic particles. ${ }^{29}$ So, considering that SEM investigates roughness and topography in different surface planes. ${ }^{30-32}$ We were able to model the possible interactions between cells and the surface of the biomaterial in relation to cytotoxicity, adhesion and biointeraction. In the case of routes where we obtain specific amounts of different calcium phosphates and obtain specific nanopowders, it was possible to identify surfaces A, B, and D with clear obtaining of the nanopowders obtained with measurements below $100 \mathrm{~nm}$, which is very important for biofunctionalization, since on surface $\mathrm{C}$, the surface was rougher and less favorable to cell biocompatibility because it was less homogeneous. Hydroxyapatite with calcium 
(a)

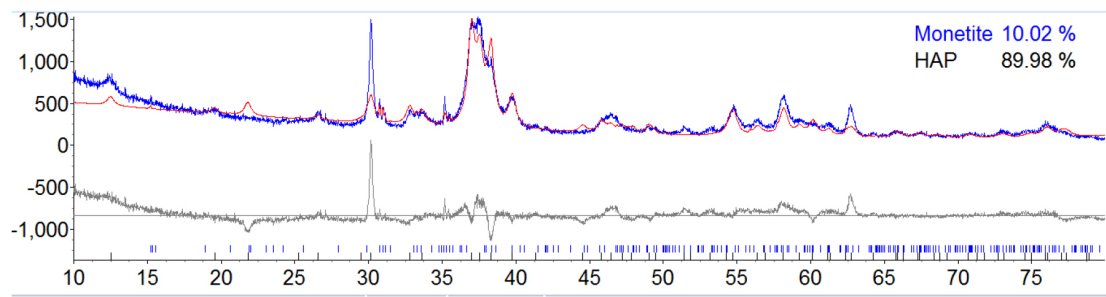

(b)

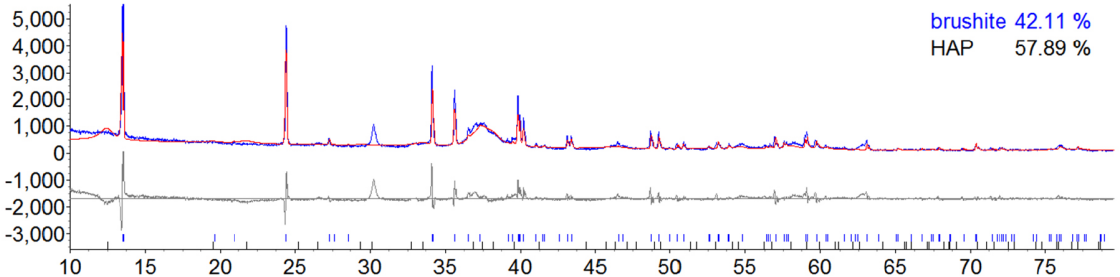

(c)

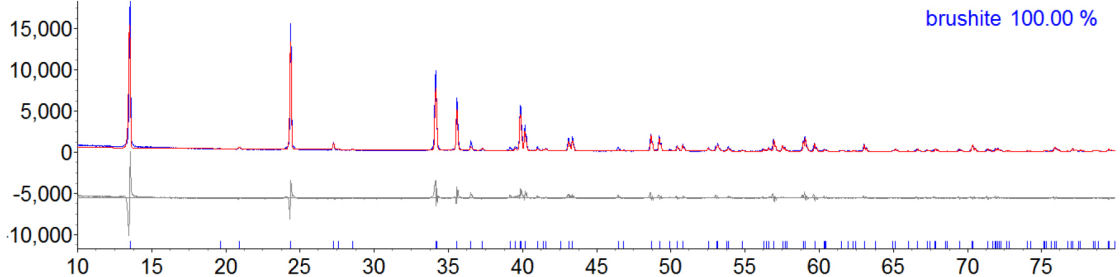

(d)

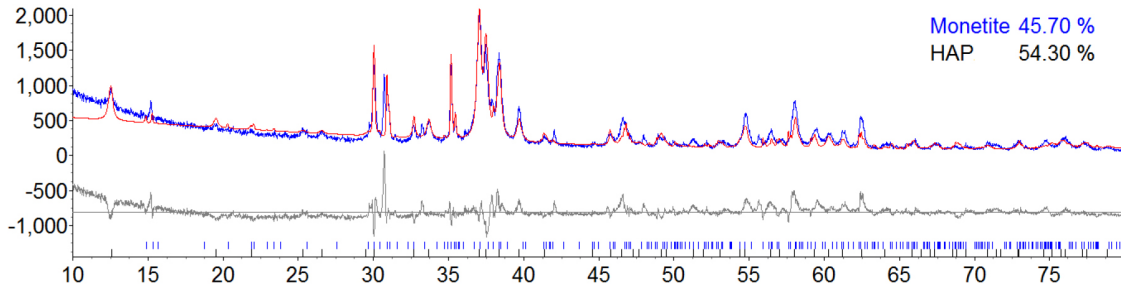

(e)

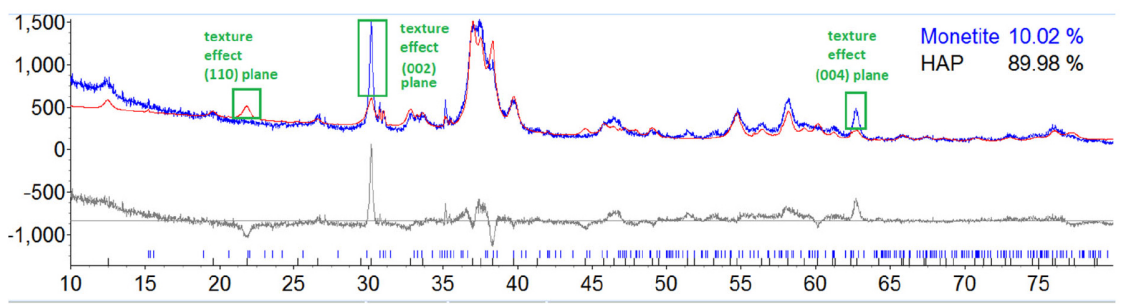

Figure 1. The XRD data after adjusting by Rietveld. The reason for some maladjustment is shown in (e)

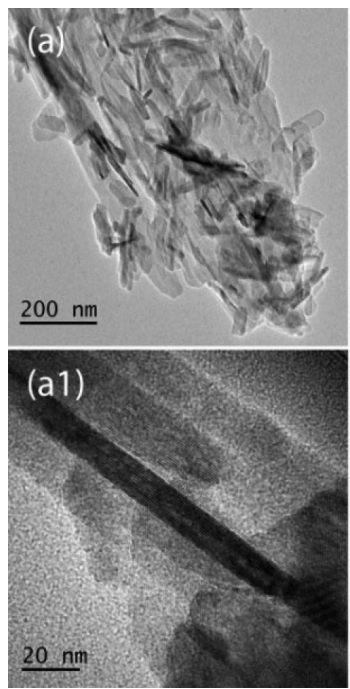

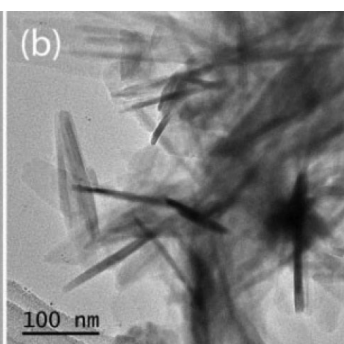

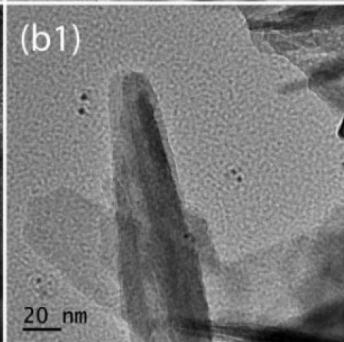

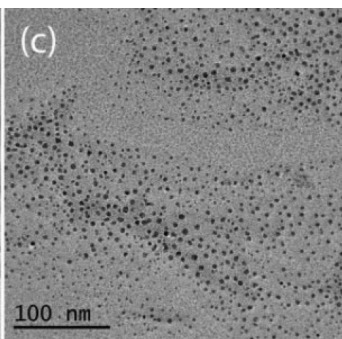
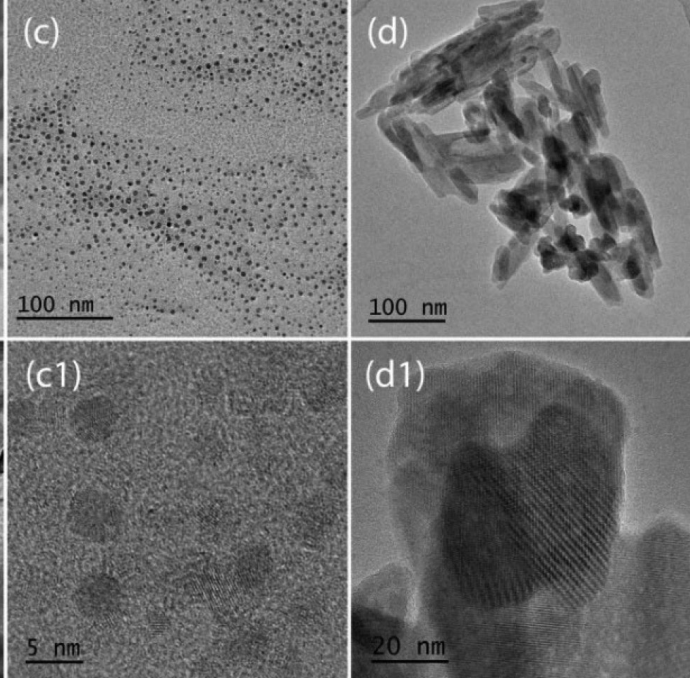

Figure 2. (a)-(d) Low-magnification TEM images of the four samples prepared by routes A-D. (a1)-(d1) High-resolution images for the corresponding samples 

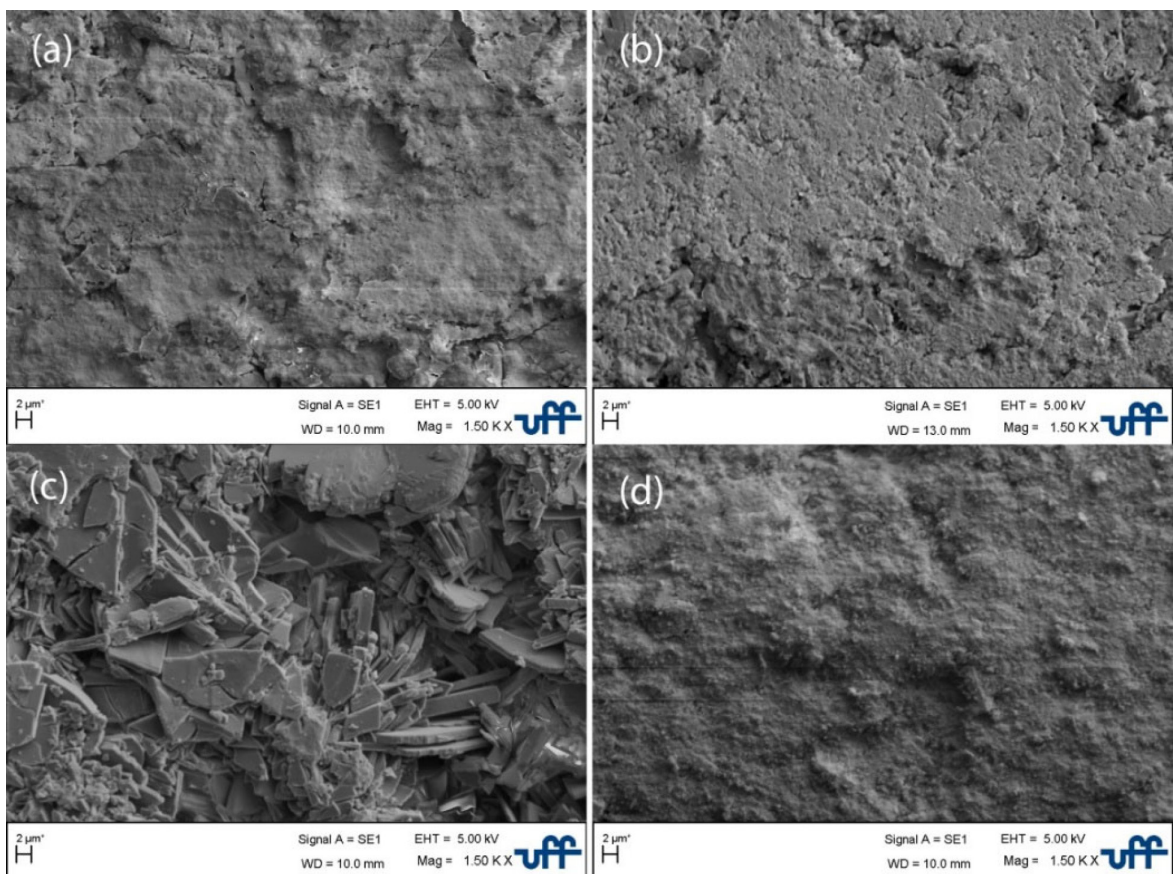

Figure 3. SEM images of the samples produced by routes A-D (Figures $3 a-3 d$, respectively)

phosphate allows for countless variations, and as a current criterion, calcium phosphate materials must exhibit anisotropic crystals or plate-like morphologies. Platelets are likewise characteristic of monetite $\left(\mathrm{CaHPO}_{4}\right)$ or brushite $\left(\mathrm{CaHPO}_{4} \cdot 2 \mathrm{H}_{2} \mathrm{O}\right){ }^{21}$

\section{Fourier-transfer infrared spectroscopy}

The results showed the identification of the classic chemical groups of the materials of the calcium phosphate group (Figure 4). More specifically, we identified hydroxyapatite in conditions a, b, and $\mathrm{c}$ and brushite in condition $\mathrm{c}$. The bands present in the FTIR spectrum show the presence of absorption of $\mathrm{H}_{2} \mathrm{O}, \mathrm{OH}^{-},\left(\mathrm{PO}_{4}\right)^{3-}, \mathrm{NO}_{3}^{-}$, and $\mathrm{CO}_{3}^{-}$ groups, which are commonly found in calcium apatites. ${ }^{33}$ Thus, the bands in regions 1033 and 1037 are identified in the group $\left(\mathrm{PO}_{4}\right)^{3-}$, which are referred to as asymmetric stretching, $v 3$ of the connections of $\mathrm{P}$ and $\mathrm{O}$ for the asymmetric stretching $v 1$. An asymmetric inflection in $v 4$ is observed in $565-566 \mathrm{~cm}^{-1}$ and $603-604 \mathrm{~cm}^{-1}$ regions. ${ }^{34}$ The $\mathrm{OH}^{-}$band appears in the 3567 and $671 \mathrm{~cm}^{-1}$ regions, which refer to the stretching symmetry mode of the hydroxyapatite structure,

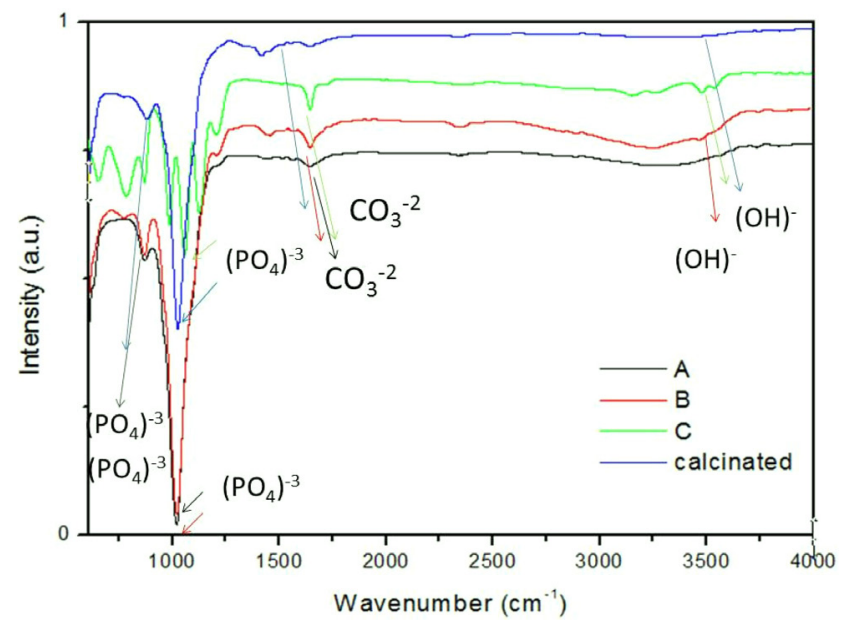

Figure. 4. Fourier-transform spectroscopy from the analyses of the different routes of hydroxyapatite calcium phosphate and the $3556 \mathrm{~cm}^{-1}$ region symbolizes the hydroxyl group as an extension and twisting vibration. ${ }^{35}$ The vibrations of the adsorption of water molecules in the hydroxyapatite structure are present in $1629-1656 \mathrm{~cm}^{-1}$ and $3430-3456 \mathrm{~cm}^{-1}$ regions. The vibrations of the $\mathrm{CO}_{3}^{2-}$ group appear between $1458-1460 \mathrm{~cm}^{-1}$ and $874.5 \mathrm{~cm}^{-1}$ regions, showing that carbon ions from an atmospheric environment replace the hydroxyapatite phosphate ions during the synthesis of the material. FTIR analysis identified a portion of the carbonate $\left(1635-1335 \mathrm{~cm}^{-1}\right)$ and the carboxyl group, which is described in the band $\left(1750-1400 \mathrm{~cm}^{-1}\right) \cdot{ }^{36}$ It also shows contributions from phosphate (1171-901 cm $\mathrm{cm}^{-1}$ and $2391 \mathrm{~cm}^{-1}$ ), similar to the literature. ${ }^{36}$ The classic slot profile of the valley of the graph expressed with 3 valleys slot with smaller size corresponds phosphate region (1000, 1050 and $1100 \mathrm{~cm}^{-1}$ ) for the shortest brushite powders analysis, while for the other hydroxyapatite with calcium phosphates obtained from the other routes (A, B and D) it presents 2 valleys and more pronounced of slot in the valley of the curve of the graph the region of $1000 \mathrm{~cm}^{-1}$ to very close. It is possible to observe relative bands of hydrogen phosphate $\left(\mathrm{HPO}_{4}{ }^{2-}\right)$ in 2391,874 , and $791 \mathrm{~cm}^{-1}$ bands and phosphate phase characteristics in 3542 and $662 \mathrm{~cm}^{-1}$ bands, which are signed in the $\mathrm{OH}^{-}$group in the structure of the crystalline phase. The phosphate group spectrum in 1031,604 , and $567 \mathrm{~cm}^{-1}$ and the carbonate bands in 1474,1421 , and $872 \mathrm{~cm}^{-1}$ confirmed the carbonate group. ${ }^{37}$

\section{Raman spectroscopy}

Using Raman analysis, we identified the peak region of hydroxyapatite with calcium phosphate from the eggshells rich in calcium carbonate using the four routes obtained. Being a nondestructive character analysis, it is possible to make analysis using other technique in the unique sample which reduces the bias, we need the sample not to be fluorescent because it this causes interference in the analysis causing an error or making the analysis impossible. As shown in Figure 5, the results showed the peak region as hydroxyapatite patterns (similar to the literature), although many of the databases and references are still being built.

RS, a form of vibrational spectroscopy, is based on the excitation of molecular vibration by electromagnetic waves. In Raman 

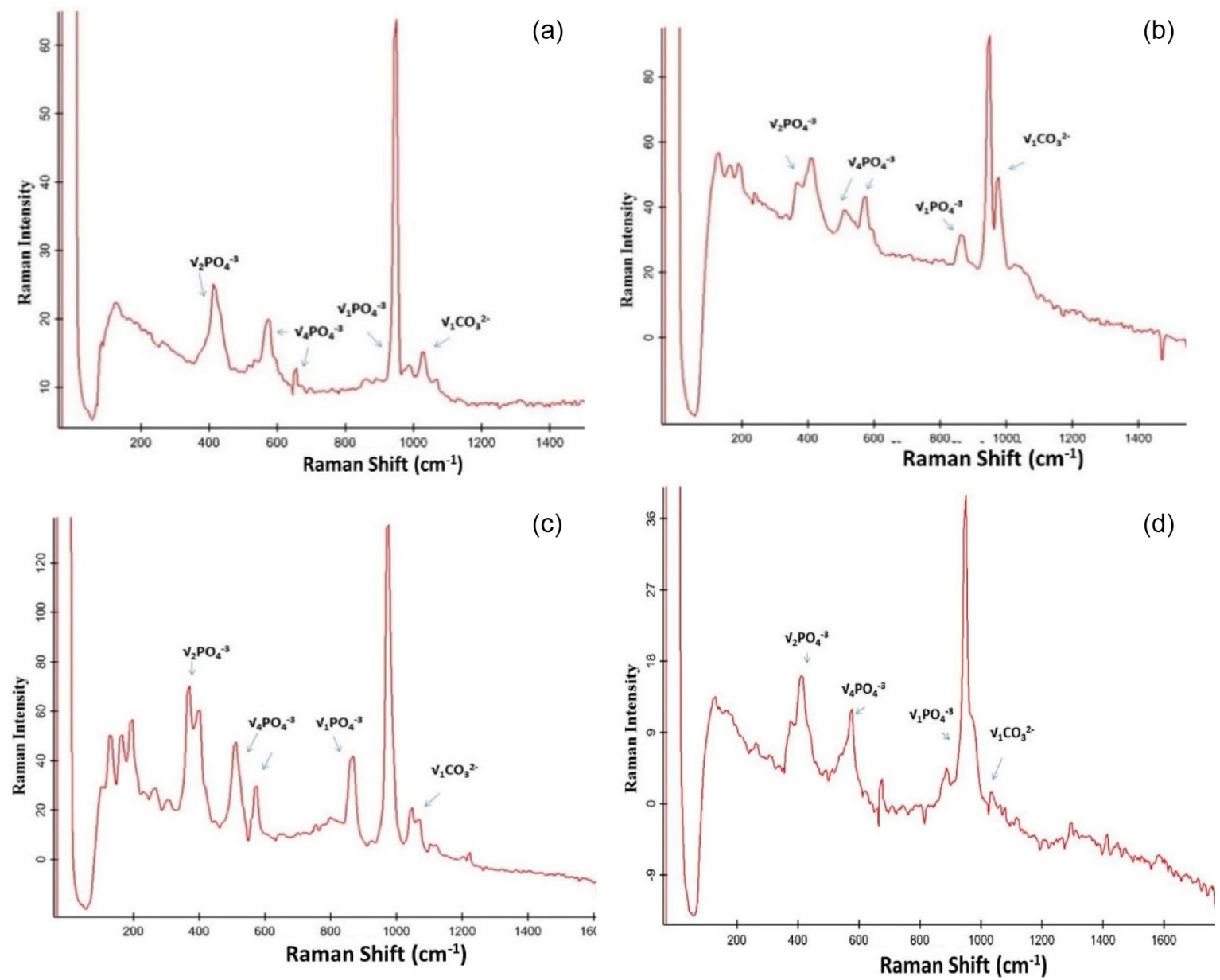

Figure 5. Raman analysis of hydroxyapatite $(a, b, d)$ and brushite (c) obtained from eggshells using the four routes

analysis, it was possible to identify specific regions that identify the specific chemical groups of hydroxyapatite with calcium phosphate as fingerprint obtained from the four routes which represent the translation of the identification of the chemical groups related to the spreading of the Raman. The main objective of these analyses is to identify the groups present in the $\mathrm{PO}_{4}{ }^{3-}, \mathrm{CO}_{3}{ }^{2-}$ in which they were identified in conditions $\mathrm{a}, \mathrm{b}$, and d. Especially in the condition of synthesis and identified as brushite by Rietveld, it was possible to identify a peculiar spectrum in the same way in Raman. For CastilhoPaz et al. in RS, the $960 \mathrm{~cm}^{-1}$ band corresponds to the vibration $v 1$ $\mathrm{PO}_{4}{ }^{36-38}$

Using FTIR and Raman analyses, we could identify the $\mathrm{CO}_{3}{ }^{2-}$ and $\mathrm{HPO}_{4}{ }^{2-}$ groups. ${ }^{39,40}$ Consequently, we could identify the anionic $\mathrm{CO}_{3}{ }^{2-}$ bands in the apatite structure, and the $\mathrm{PO}_{4}{ }^{3-}$ band also assigns $1030,1048,960,587$, and $431 \mathrm{~cm}^{-1}$ bands to the $\mathrm{PO}_{4}{ }^{3-}$ and $1073 \mathrm{~cm}^{-1}$ band to the $\mathrm{CO}_{3}{ }^{2-} \cdot{ }^{38}$ Carbonated nanocrystalline hydroxyapatite was evaluated by Raman using $2-\mathrm{cm}^{-1}$ resolution in a range from 200 to $4000 \mathrm{~cm}^{-1}$ using 30 scans. Thus, the spectra in the studies of Ag-hydroxyapatite compared with pure hydroxyapatite showed the 429 and $450 \mathrm{~cm}^{-1}$ bands ( $v 2 \mathrm{PO}_{4}{ }^{3-}$ ), showing the $961 \mathrm{~cm}^{-1}$ band $\left(v 1 \mathrm{PO}_{4}^{3-}\right)$ being assigned with typical of the phosphate band associated with apatite carbonate. ${ }^{39}$

Finally, the 1045 and $1075 \mathrm{~cm}^{-1}$ bands $\left(\mathrm{v} \mathrm{PO}_{4}{ }^{3-}\right)$ are associated with vibrational stretching modes in another study where RS was used to evaluate hydroxyapatite in the dental composite. In this case, phosphate peaks are assigned in 901, 912, and $1108 \mathrm{~cm}^{-1}$ bands, in addition to 943 and $968 \mathrm{~cm}^{-1}$ peaks, in the case that is assigned to the $\beta$-TCP one of the most soluble calcium phosphates closest to the brushite and this phase when present in the case that the occurrence of the $\beta$-TCP phase occurs after immersion in blood plasma simulating fluid (SBF), with the formation of the phase and a more intense peak in the $960 \mathrm{~cm}^{-1}$ band assigned, which is more intentional and evidenced. ${ }^{40}$ Raman analysis has been applied to countless studies in bone engineering as it identifies the phases of mineralization, detects changes in the differentiation of primary cell cultures, and quantifies changes in matrix mineralization. ${ }^{41,42}$

\section{Thermogravimetric analysis}

Using TGA analysis, we verified that regardless of the route, the mass loss occurred at $\sim 200{ }^{\circ} \mathrm{C}$, as shown in Figure 6. It observed a trend of small mass gain in the temperature around $150{ }^{\circ} \mathrm{C}$ (in the brushite $\mathrm{C}$-route experiment). In the first quartile of the programmed heating ramp flow path until it reaches a temperature of $800^{\circ} \mathrm{C}$ which visualizes a loss of mass of $600{ }^{\circ} \mathrm{C}$ in the graph.

The powders' characterization obtained by the four routes made it possible to identify the mass loss profile region typically of hydroxyapatites and brushite (route $\mathrm{C}$ ). The thermal analysis performed was the TGA (thermogravimetric analyses were performed on the powder obtained from the syntheses), obtaining the green powders from the precursors and after drying and stoving for $50{ }^{\circ} \mathrm{C}$ overnight. At a temperature of $50{ }^{\circ} \mathrm{C}$ on the graph there was a progressive loss of mass gradually decreasing until reaching $200{ }^{\circ} \mathrm{C}$ where occurred to the maximum mass loss slot. The the maximum loss of HA with calcium phosphate of the materials in green at $200{ }^{\circ} \mathrm{C}$ suggests an endothermic effect associated with water loss and the low temperature being as the curve slot for calcium phosphates suggests the presence of low crystallinity materials. 


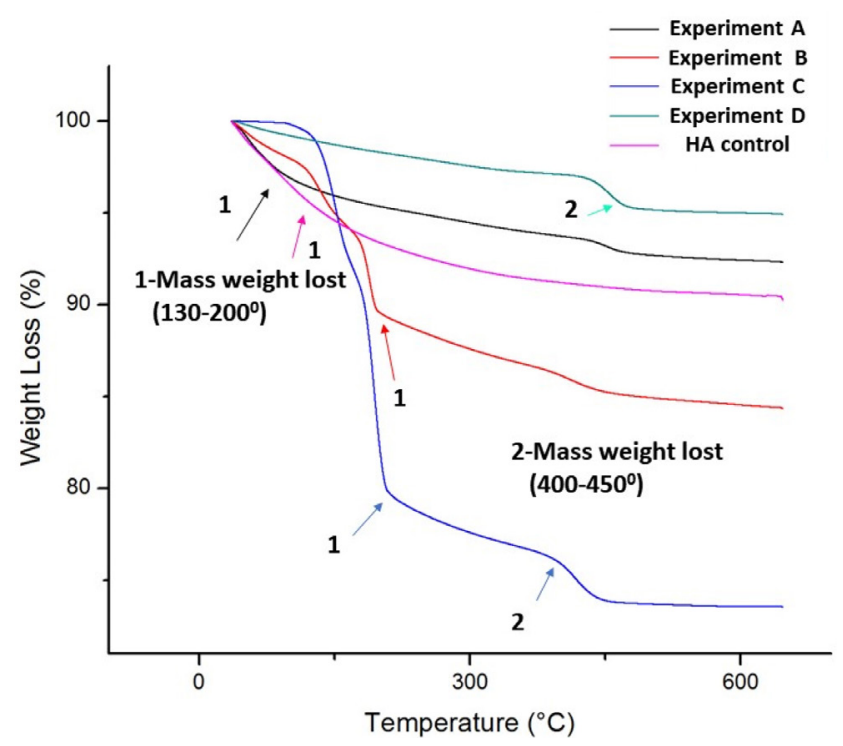

Figure 6. Analysis of the TGA obtained from the four routes of the eggshell HA

Between $200{ }^{\circ} \mathrm{C}$ and $500{ }^{\circ} \mathrm{C}$, the mass decreased dramatically. It refers to a characteristic endothermic event that may be associated with the loss of hydration water. The TGA cycle used varied from $0{ }^{\circ} \mathrm{C}$ to $800{ }^{\circ} \mathrm{C}$ and the maximum mass loss expressed in curve is is represented $0-600{ }^{\circ} \mathrm{C} . .^{43}$ The mass loss above $400{ }^{\circ} \mathrm{C}-450$ ${ }^{\circ} \mathrm{C}$ is probably because of the greater crystallinity of the apatite and carbonate loss. Thus, an increase in mass may be associated with an endothermic phenomenon, whereas mass loss with an exothermic phenomenon is related to small structural arrangements and oxidation reactions

\section{CONCLUSIONS}

We evaluated four routes for the production of hydroxyapatite. HA was obtained in three of the routes, and one of them, it was obtained brushite. Results were compared using different analyses. This study of nanoscale materials and new powders' synthesis routes requires different stoichiometric accessories used in the synthesis technique and new specific controlled steps to obtain nanopowders. Thus, it was possible to predict different compounds and identify and compare the different samples on the nanoscale with varying sizes, forming about $100 \mu \mathrm{m}$ [in the form of crystallite (hydroxyapatite and monetite)]. So, route A and B by TEM with a length of higher than $100 \mathrm{~nm}$ and a width of $20 \mathrm{~nm}$. Rietveld analysis presented monoclic particles and crystal sizes of $18 \mathrm{~nm}(\mathrm{~A}), 15 \mathrm{~nm}$ (B) and D (40 nm). It is also possible to describe the brushite with a structure of greater symmetry because Curry and Jones ${ }^{26}$ suggested that it is a phase of less symmetry. It presented structural data where the produced brushite was found to be of structure SG = $15 \mathrm{I} 2 / \mathrm{a}$. There are several different monetites in the literature; however, only one could "stare" at the monetite of its spectra. The topographic analysis by SEM of powders built in tablets showed a good future perspective of applying on surface which allows good surface biofunctionalization in biomaterials in A, B and D routes, indicating a good perspective to cell application in the future study. Thermal analysis showed that the identification of mass loss was more evident in route $\mathrm{C}$ (brushite) with slot at $200{ }^{\circ} \mathrm{C}$ that suggest low cristalinity. The compound powders were identified by chemical analysis by the fingerprint and by detecting the chemical elements present, including building a Raman database, mainly the key chemical identification groups as $\left(\mathrm{PO}_{4}\right)^{3-}$ and $\left(\mathrm{CO}_{3}^{-}\right)$. It was possible to obtain the biofunctionalized powders by the routes proposed.

\section{ACKNOWLEDGMENTS}

IR de Lima would like to thank CAPES/PNPD for financial support. MF de Campos, JA de Castro, and GS Fonseca thank FAPERJ and CNPq. The authors thank the financial support from the Metallurgical and Materials Engineering Programs at UFF (Volta Redonda). The authors also thank the COPPE/UFRJ and LaMAR/ UFF for their support and analysis of the characterization. The authors declare no conflict of interest.

\section{REFERENCES}

1. Eliaz, N.; Metoki, N.; Materials 2017, 10, 334.

2. Teles, A. L. B; dos Santos Júnior, M. C; Braga, V. M. L; Orbital: Electron. J. Chem. 2015, 7, 292.

3. Schneider, N.; Subkowski, T.; Jenewein, K. M; Bollscweiler, W. V., Henkes, T.; Facey, Hauer, B., (2018), Bonding or bleaching methods to the enamel surface and / or dentin, repair or inhibition of dental erosion, promotion of dental remineralization and / or intensification of the fluoride anti-cavity effects and production of an oral care product comprising dentifrice HABP, use of HABP in the manufacture of an oral care composition comprising HABP, hydroxyapatite-binding polypeptide and chewing gum composition, National patent application filed. Order number BR1120180082210A2.

4. Ferraris, S.; Yamaguchi, S.; Barbani, N.; Cazzola, M.; Cristallini, C.; Miola, M.; Vernê , E.; Spriano S.; Acta Biomater. 2020, 102, 468.

5. Kharissova, O. V.; Kharisov, B.; González, C. M. O.; Mendez, Y. P. López, I.; R. Soc. Open Sci. 2019, 6, 191378.

6. Abidi, S. A. A.; Murtaza, Q.; J. Mater. Sci. Technol. 2014, 30, 307.

7. Baltazar-Ruiz, A. J; Reyes-López, S.Y; Silva-Holguin, P. N; Larranaga, D.; Estévez M. P. R.; Results Phys. 2018, 7, 2639.

8. Triyono, J.; Alfiansyah, R.; Sukanto, H.; Ariawan, D.; Nugroho, Y.; Bioprinting 2020, 18, 35 .

9. Maidaniuc, A.; Miculescu, F.; Voicu, S. I; Andronescu, C.; Ciocan, L.T.; Appl. Surf. Sci. 2018, 438, 158.

10. Zhang, H.; Liu, H.; Yue, J.; 2014, Chem. Rev. 2014, 1, 114

11. Vemuri, S.; Abraham, S.; Azamthulla, M.; Furtado, S.; Bharath S.; Wound Medicine 2020, 28, 100177.

12. Bee, S. L; Mariatti, M.; Ahmad, N.; Yahaya, B. H.; Hamid Z. A. A; Mater. Today: Proc. 2019, 16, 1876.

13. Barua, E.; Deoghare A. B; Deb, P., Lala, S. D; Mater. Today: Proc. 2019, $15,188$.

14. Li, M.; Xiong, P.; Yan, F.; Li, S.; Ren, C.; Yin, Z.; Li, A.; Li., H.; Ji, X.; Zheng, Y.; Cheng, Y.; X. P.; Van, F.; Li, S.; Ren, C.; Yin, Z.; Bioact. Mater. 2018, 3, 18.

15. Farias, K. A. S; Sousa, W. J. B; Cardoso, M. J. B; Lima, R. J. S; Rodriguez, M. A; Fook, M. V. L; Cerâmica 2019, 65, 99.

16. Martinez, C.; Krieke, G.; Locs, J.; Cimdina, B.; Garrido, L.; Gilabert, U.; Ozols A.; Procedia Mater. Sci. 2015, 8, 324.

17. Youness, R.; Mohamed A. T.; Ehaes, H.; Medhat, I.; Mater. Chem. Phys. 2017, 190, 209.

18. Ferraris, S.; Yamaguchi, S.; Barbani, N.; Cazzola, M.; Cristallini, C.; Miola, M.; Vernê, E.; Spriano, S.; Acta Biomater. 2020, 102, 468.

19. Stammeier, J. A.; Purgstaller, B.; Hippler, D.; Mavromatis, V.; Dietzel, M.; MethodsX 2018, 5, 1241.

20. Teixeira, A. M. R. F.; Ogasawra, T.; Nóbrega, M. C. S.; 2006, Mater. Res. 2006, 9, 3.

21. Drouet C.; BioMed Res. Int. 2013, 4, 490946.

22. Kuznetsov, V. N; Yanovska, A. A.; Stanislavov, A. S.; Danilchenko, S. N.; Kalinkevich, A. N.; Sukhodub, L. F.; Mater. Sci. Eng., C 2016, 60, 547.

23. Dosen, A.; Giese, R. F.; Am. Mineral. 2011, 96, 368.

24. Posner, A.; Perloff, A.; Alfred, F.; Acta Crystallogr. 1958, 11, 308. 
25. MacLennan, G.; Beevers, C. A.; Acta Crystallogr. 1955, 8, 579.

26. Curry, N. A; Jones, D. W.; J. Chem. Soc. A 1971, 3725.

27. Díaz-Sainz, C. I.; Villacampa, A.; Otálora, F.; Am. Mineral. 2004, 89, 307.

28. Lijima, K.; Suzuki, R.; Lizuka, A.; Hashizume, M.; Colloids Surf., B 2016, 147, 351 .

29. Vahdat, A.; Ghasemi, B.; Yousefpour, M.; S. Afr. J. Chem. Eng. 2020, $33,90$.

30. Nikara, S.; Ahmadi, E.; Nia, A.; A.; Journal of Agriculture and Food Research 2020, 2, 100036.

31. Goggin, P.; Ho, E. M. L.; Gnaegi, H.; Searle, S.; Oreffo, R. O. C.; Bone 2020, 131, 115107.

32. Maraghechi, S.; Hoefnagels, J. P. M.; Peerlings, R. H. J; Geers, M. G. D.; Ultramicroscopy 2018, 187, 144.

33. Goldberg, M. A.; Protsenko, P. V.; Smirnov, V. V.; Antonova, O. S.; Smirnov, S. V.; Konovalov, A. A.; Vorckachev, K. G.; Kudryavtsev, E. A.; Barinov, S. M.; Komlev, V. S.; J. Mater. Res. Technol. 2020, 9, 76.

34. Zhang, H. Liu.; H.; Yue, J.; Chem. Rev. 2014, 114, 88.
35. Sossa, P. A. F.; Giraldo, B. S.; Garcia, B. C. G.; Matéria 2018, 23, 4.

36. Boavenntura, T. P.; Peres, A. M.; Gil, V. S. B.; Gil, C. S. B.; Quim. Nova 2020, 43, 2.

37. Senra, M. R.; de Lima R. B.; Souza, D. h. S.; Marques, M. F.; Monteiro, S. N.; J. Mater. Res. Technol. 2020, 9, 190.

38. Castilho-Paz, A. M.; Londono-Restrepo, S. M.; Tirado-Meíja, L.; Mondragon, M. A.; Rodriguez-García, M. E.; Prog. Nat. Sci.: Mater. Int. 2020, 30, 494.

39. Pecheva, E. V., Pramatarova, L. D.; Maitz, M. F.; Pham, M. T.; Kondyuirin, A. V.; Appl. Surf. Sci. 2004, 235, 176.

40. Dosen, A.; Giese, R. F.; Am. Mineral. 2011, 96, 368.

41. Goloshchapov, D. L.; Ippolitov, Y. A.; Seredin, P. V.; Results Phys. 2020, $18,103277$.

42. Aljabo, A.; Neel, E. A. A.; Knowles, J. C.; Young, A. M.; Mater. Sci. Eng., C 2016, 60, 285.

43. Santos, M. L.; Florentino, A. O.; Saeki, M. J.; Aparecida, A. H.; Fook, M. V. L.; Guastaldi, A.; Ecletica Quim. 2005, 30, 3. 